\title{
The periodic rotary motions of a rigid body in a new domain of angular velocity
}

\author{
A. I. Ismail ${ }^{1,2^{*}}$ (1)
}

*Correspondence:
aiismail@uqu.edu.sa;
abdelazeez-esmaeel@science.
tanta.edu.eg
${ }^{1}$ Mechanical Engineering
Department, College
of Engineering and Islamic
Architecture, Umm Al-Qura
University, P. O. Box 5555, Makkah,
Saudi Arabia
Full list of author information
is available at the end of the
article

*Correspondence: aiismail@uqu.edu.sa abdelazeez-esmaeel@science. tanta.edu.eg ${ }^{1}$ Mechanical Engineering Department, College of Engineering and Islam University, P. O. Box 5555, Makkah, Saudi Arabia is available at the end of the

\begin{abstract}
In the previous works, the limiting case for the motion of a rigid body about a fixed point in a Newtonian force field, which comes from a gravity center lies on Z-axis, is solved. The authors apply the small parameter technique which is achieved giving the body a sufficiently large angular velocity component $r_{0}$ about the fixed $z$-axis of the body. The periodic solutions of motion are obtained in neighborhood $r_{0}$ tends to $\infty$. In our work, we aim to find periodic solutions to the problem of motion in the neighborhood of $r_{0}$ tends to 0 . So, we give a new assumption that: $r_{0}$ is sufficiently small. Under this assumption, we must achieve a large parameter and search for another technique for solving this problem. This technique is named; a large parameter technique instead of the small one well known previously. We see the advantage of the new technique which appears in saving high energy used to begin the motion and give the solution of the problem in another domain. The obtained solutions by the new technique depend on $r_{\mathrm{o}}$. We consider that the center of mass of this body does not necessarily coincide with the fixed point $\mathrm{O}$. We reduce the six nonlinear differential equations of the body and their three first integrals to a quasilinear autonomous system of two degrees of freedom and one first integral. We solve the rational case when the frequencies of the generating system are rational except ( $\omega=1,2,1 / 2,3,1 / 3, \ldots)$ under the condition $\gamma_{0}^{\prime \prime}=\cos \theta_{0} \approx 0$. We use the fourth-order Runge-Kutta method to find the periodic solutions in the closed interval of the time $t$ and to compare the analytical method with the numerical one.
\end{abstract}

Keywords: Equations of Euler, Rigid body dynamics, Mathematical techniques, Gyroscopic motions, Numerical methods, Satellite motions, Navigation

Mathematics Subject Classification: 70E05, 70E15, 70E17, 70E20

\section{Introduction}

Some asymptotic perturbed techniques [1-3] are widely used by many authors for solving the ordinary linear and nonlinear systems for differential equations in different problems of engineering, mathematical physics, and astronomy. As an extension of this type of problem, we use some perturbation and numerical techniques in the movement of coherent bodies around a fixed point in the presence of new conditions to obtain periodic solutions of different scales to those obtained before.

As in [4], let the fixed $z$-axis of the ellipsoid of inertia of the body makes an angle $\theta_{o} \approx \pi / 2$ with the fixed $Z$-axis in space. We assume the new value of $r_{\mathrm{o}}$, which is author(s) and the source, provide a link to the Creative Commons licence, and indicate if changes were made. The images or other third party material in this article are included in the article's Creative Commons licence, unless indicated otherwise in a credit line to the material. If material is not included in the article's Creative Commons licence and your intended use is not permitted by statutory regulation or exceeds the permitted use, you will need to obtain permission directly from the copyright holder. To view a copy of this licence, visit http:// creativecommons.org/licenses/by/4.0/. 
sufficiently small instead of sufficiently large value in [4]. We define a large parameter $\mu$ proportional to $1 / r_{\mathrm{o}}$ instead of the small one in [4]. Consider A, B, C represent the moments of inertia of the body, $\mathrm{p}, \mathrm{q}, \mathrm{r}$ are the components of the angular velocity vector and $\gamma, \gamma^{\prime}, \gamma^{\prime \prime}$ are the direction cosines of the unit vector in direction of the $Z$-axis. So, the equations of motion and their three first integrals are derived in the form:

$$
\begin{aligned}
& \dot{p}_{1}+A_{1} q_{1} r_{1}=\mu^{-1} a^{-1}\left(\gamma^{\prime \prime} y_{0}^{\prime}-\gamma^{\prime} z_{0}^{\prime}+k a A_{1} \gamma^{\prime} \gamma^{\prime \prime}\right), \\
& \dot{q}_{1}+B_{1} p_{1} r_{1}=\mu^{-1} b^{-1}\left(\gamma z_{0}^{\prime}-\gamma^{\prime \prime} x_{0}^{\prime}+k b B_{1} \gamma^{\prime \prime} \gamma\right), \\
& \dot{r}_{1}=\mu^{-2}\left(-C_{1} p_{1} q_{1}+\gamma^{\prime} x_{0}^{\prime}-\gamma y_{0}^{\prime}+k C_{1} \gamma \gamma^{\prime}\right), \\
& \dot{\gamma}=r_{1} \gamma^{\prime}-\mu^{-1} q_{1} \gamma^{\prime \prime}, \quad \dot{\gamma}^{\prime}=\mu^{-1} p_{1} \gamma^{\prime \prime}-r_{1} \gamma, \quad \dot{\gamma}^{\prime \prime}=\mu^{-1}\left(q_{1} \gamma-p_{1} \gamma^{\prime}\right) . \\
& r_{1}^{2}=1+\mu^{-2} s_{1}, \quad r_{1} \gamma^{\prime \prime}=\gamma_{0}^{\prime \prime}+\mu^{-1} s_{2}, \quad \gamma^{2}+\gamma^{\prime 2}+\gamma^{\prime \prime 2}=1 .
\end{aligned}
$$

Such that:

$$
\begin{aligned}
s_{1} & =s_{3}-2 z_{0}^{\prime}\left(\gamma_{0}^{\prime \prime}-\gamma^{\prime \prime}\right)+k\left(\gamma_{0}^{\prime \prime 2}-\gamma^{\prime \prime 2}\right), \\
s_{2} & =a\left(p_{10} \gamma_{0}-p_{1} \gamma\right)+b\left(q_{10} \gamma_{0}^{\prime}-q_{1} \gamma^{\prime}\right) \\
s_{3} & =a\left(p_{10}^{2}-p_{1}^{2}\right)+b\left(q_{10}^{2}-q_{1}^{2}\right)-2\left[x_{0}^{\prime}\left(\gamma_{0}-\gamma\right)+y_{0}^{\prime}\left(\gamma_{0}^{\prime}-\gamma^{\prime}\right)\right] \\
& +k\left[a\left(\gamma_{0}^{2}-\gamma^{2}\right)+b\left(\gamma_{0}^{\prime 2}-\gamma^{\prime 2}\right)\right] . \\
p_{1} & =p / c, \quad(p q r), \quad \gamma_{0}>0, \quad \mu=c / r_{0}, \quad l^{2}=x_{0}^{2}+y_{0}^{2}+z_{0}^{2}, \\
k & =N / c^{2}, \quad A_{1}=(C-B) / A, \quad(A B C) .
\end{aligned}
$$

\section{Reduction of the equations of motion to a quasilinear autonomous system}

Solving the first and the second equations of (2) for $r_{1}$ and $\gamma^{\prime \prime}$, we get:

$$
r_{1}=1+\frac{1}{2} \mu^{-2} s_{3}+\ldots, \quad \gamma^{\prime \prime}=\gamma_{0}^{\prime \prime}+\mu^{-1} s_{2}-\frac{1}{2} \mu^{-2} s_{3} \gamma_{0}^{\prime \prime}+\ldots
$$

Differentiating the first and the fourth equations of (1) and using (6) for reduction in the four remaining equations into two differential equations of the second order, we get:

$$
\begin{aligned}
\ddot{p}_{1} & +\omega^{2} p_{1}=\mu^{-1}\left[z_{0}^{\prime}\left(a^{-1}-A_{1} b^{-1}\right) \gamma+A_{1} b^{-1} x_{0}^{\prime} \gamma_{0}^{\prime \prime}-k\left(A_{1}-\omega^{2}\right) \gamma_{0}^{\prime \prime} \gamma\right] \\
& +\mu^{-2}\left\{A_{1} x_{0}^{\prime}\left(b^{-1} s_{2}-q_{1} \gamma^{\prime}\right)-\omega^{2} p_{1} s_{3}+p_{1}\left(A_{1} C_{1} q_{1}^{2}-a^{-1} z_{0}^{\prime} \gamma_{0}^{\prime \prime}\right)\right. \\
& +y_{0}^{\prime}\left[\left(2 A_{1}+a^{-1} b\right) q_{1} \gamma-a^{-1} p_{1} \gamma^{\prime}\right]+k A_{1}\left[p_{1}\left(\gamma_{0}^{\prime \prime 2}-\gamma^{\prime 2}\right)\right. \\
& \left.\left.+q_{1}\left(1-C_{1}\right) \gamma \gamma^{\prime}-s_{2}\left(1+B_{1}\right) \gamma\right]\right\}+\mu^{-3}\left\{0.5\left(a^{-1}-A_{1} b^{-1}\right) z_{0}^{\prime} s_{3} \gamma\right. \\
& \left.-s_{2} p_{1}\left[z_{0}^{\prime}\left(2 \omega^{2}+a^{-1}\right)-2 k\left(A_{1}+\omega^{2}\right) \gamma_{0}^{\prime \prime}\right]\right\}+\ldots \\
\ddot{\gamma} & +\gamma=\mu^{-1} \gamma_{0}^{\prime \prime} p_{1}\left(1+B_{1}\right)+\mu^{-2}\left\{p_{1}\left[\left(1+B_{1}\right) s_{2}+\left(1-C_{1}\right) q_{1} \gamma^{\prime}\right]\right. \\
& -\gamma\left[s_{3}+y_{0}^{\prime} \gamma^{\prime}+z_{0}^{\prime} b^{-1} \gamma_{0}^{\prime \prime}+q_{1}^{2}+k\left(C_{1} \gamma^{\prime 2}-B_{1} \gamma_{0}^{\prime \prime 2}\right)\right] \\
& \left.+x_{0}^{\prime}\left(b^{-1} \gamma_{0}^{\prime \prime 2}+\gamma^{\prime 2}\right)\right\}+\mu^{-3}\left\{2 b^{-1} x_{0}^{\prime} \gamma_{0}^{\prime \prime} s_{2}\right. \\
& \left.+\left[2 k\left(1-B_{1}\right) \gamma_{0}^{\prime \prime}-z_{0}^{\prime}\left(2+b^{-1}\right)\right] s_{2} \gamma_{2}\right\}+\ldots
\end{aligned}
$$

Here: 


$$
\omega^{2}=-A_{1} B_{1}=(A-C)(B-C) / A B=(a-1)(b-1) / a b .
$$

Solving the first and the fourth equations of the system (1) and using (6), we get:

$$
\begin{aligned}
& q_{1}=-\dot{p}_{1} A_{1}^{-1}+\mu^{-1} a^{-1} A_{1}^{-1}\left(y_{0}^{\prime} \gamma_{0}^{\prime \prime}-z_{0}^{\prime} \gamma^{\prime}+a k A_{1} \gamma^{\prime} \gamma_{0}^{\prime \prime}\right)+\ldots, \\
& \gamma^{\prime}=\dot{\gamma}-\mu^{-1} A_{1}^{-1} \gamma_{0}^{\prime \prime} \dot{p}_{1}+\ldots
\end{aligned}
$$

Introducing the new variables $p_{2}$ and $\gamma_{2}$ such that:

$$
\begin{aligned}
& p_{1}=p_{2}+\mu^{-1} \chi_{1} \gamma_{2}+\mu^{-1} \frac{x_{0}^{\prime}}{1-a} \gamma_{0}^{\prime \prime}, \\
& \gamma=\gamma_{2}+\mu^{-1} a \gamma_{0}^{\prime \prime} p_{2},
\end{aligned}
$$

where:

$$
\chi_{1}=\left(1-\omega^{2}\right)^{-1}\left[-z_{0}^{\prime}\left(a^{-1}-A_{1} b^{-1}\right)+k \gamma_{0}^{\prime \prime}\left(A_{1}-\omega^{2}\right)\right] .
$$

Substituting from (9) into (8), we get:

$$
\begin{aligned}
& q_{1}=-A_{1}^{-1} \dot{p}_{2}+\mu^{-1} A_{1}^{-1}\left(y_{0}^{\prime} a^{-1} \gamma_{0}^{\prime \prime}-\chi_{2} \dot{\gamma}_{2}\right)+\ldots, \\
& \gamma^{\prime}=\dot{\gamma}_{2}-\mu^{-1} \gamma_{0}^{\prime \prime} A_{1}^{-1} b \dot{p}_{2}+\ldots, \quad \chi_{2}=\chi_{1}+z_{0}^{\prime} a^{-1}-k A_{1} \gamma_{0}^{\prime \prime} .
\end{aligned}
$$

Substituting (10) and (9) into (3), we get:

$$
s_{i}=s_{i 1}+2^{2-i} \mu^{-1} s_{i 2}+\ldots, \quad i=1,2,
$$

where:

$$
\begin{aligned}
s_{11} & =a\left(p_{20}^{2}-p_{2}^{2}\right)+b\left(\dot{p}_{20}^{2}-\dot{p}_{2}^{2}\right) / A_{1}^{2}-2\left[x_{0}^{\prime}\left(\gamma_{20}-\gamma_{2}\right)\right. \\
& \left.+y_{0}^{\prime}\left(\dot{\gamma}_{20}-\dot{\gamma}_{2}\right)\right]+k\left[a\left(\gamma_{20}^{2}-\gamma_{2}^{2}\right)+b\left(\dot{\gamma}_{20}^{2}-\dot{\gamma}_{2}^{2}\right)\right], \\
s_{12} & =a\left[\frac{x_{0}^{\prime}}{1-a} \gamma_{0}^{\prime \prime}\left(p_{20}-p_{2}\right)+\chi_{1}\left(p_{20} \gamma_{20}-p_{2} \gamma_{2}\right)\right] \\
& -b A_{1}^{-2}\left[y_{0}^{\prime} a^{-1} \gamma_{0}^{\prime \prime}\left(\dot{p}_{20}-\dot{p}_{2}\right)-\chi_{2}\left(\dot{p}_{20} \dot{\gamma}_{20}-\dot{p}_{2} \dot{\gamma}_{2}\right)\right] \\
& -a x_{0}^{\prime} \gamma_{0}^{\prime \prime}\left(p_{20}-p_{2}\right)+y_{0}^{\prime} b A_{1}^{-1} \gamma_{0}^{\prime \prime}\left(\dot{p}_{20}-\dot{p}_{2}\right)+\left(z_{0}^{\prime}-k \gamma_{0}^{\prime \prime}\right) s_{21} \\
& +k \gamma_{0}^{\prime \prime}\left[a^{2}\left(p_{20} \gamma_{20}-p_{2} \gamma_{2}\right)-b^{2} A_{1}^{-1}\left(\dot{p}_{20} \dot{\gamma}_{20}-\dot{p}_{2} \dot{\gamma}_{2}\right)\right], \\
s_{21} & =a\left(p_{20} \gamma_{20}-p_{2} \gamma_{2}\right)-b A_{1}^{-1}\left(\dot{p}_{20} \dot{\gamma}_{20}-\dot{p}_{2} \dot{\gamma}_{2}\right), \\
s_{22} & =a\left[a \gamma_{0}^{\prime \prime}\left(p_{20}^{2}-p_{2}^{2}\right)+\frac{x_{0}^{\prime}}{1-a} \gamma_{0}^{\prime \prime}\left(\gamma_{20}-\gamma_{2}\right)+\chi_{1}\left(\gamma_{20}^{2}-\gamma_{2}^{2}\right)\right] \\
& +b A_{1}^{-1}\left[b A_{1}^{-1} \gamma_{0}^{\prime \prime}\left(\dot{p}_{20}^{2}-\dot{p}_{2}^{2}\right)+y_{0}^{\prime} a^{-1} \gamma_{0}^{\prime \prime}\left(\dot{\gamma}_{20}-\dot{\gamma}_{2}\right)-\chi_{2}\left(\dot{\gamma}_{20}^{2}-\dot{\gamma}_{2}^{2}\right)\right], \\
s_{3} & =s_{11}+2 \mu^{-1}\left(s_{12}-z_{0}^{\prime} s_{21}+k s_{21} \gamma_{0}^{\prime \prime}\right)+\ldots .
\end{aligned}
$$

Substituting (11), and (12) into (6) yields:

$$
r_{1}=1+\frac{1}{2} \mu^{-2} s_{11}+\ldots, \quad \gamma^{\prime \prime}=\gamma_{0}^{\prime \prime}+\mu^{-1} s_{21}+\mu^{-2} s_{22}-\frac{1}{2} \mu^{-2} s_{11} \gamma_{0}^{\prime \prime}+\ldots
$$

Substituting (9), (10), (11), and (12) into (7), we obtain a quasilinear autonomous system [5]: 
$\ddot{p}_{2}+\omega^{2} p_{2}=\mu^{-2} F\left(p_{2}, \dot{p}_{2}, \gamma_{2}, \dot{\gamma}_{2}, \varepsilon\right), \quad \ddot{\gamma}_{2}+\gamma_{2}=\mu^{-2} \Phi\left(p_{2}, \dot{p}_{2}, \gamma_{2}, \dot{\gamma}_{2}, \varepsilon\right)$,

where:

$$
\begin{aligned}
& F=F_{2}+\mu^{-1} F_{3}+\ldots, \quad \Phi=\Phi_{2}+\mu^{-1} \Phi_{3}+\ldots, \\
& F_{2}=f_{2}-a \gamma_{0}^{\prime \prime} \chi_{1}\left(1-\omega^{2}\right) p_{2}, \Phi_{2}=\varphi_{2}+a \gamma_{0}^{\prime \prime}\left(1-\omega^{2}\right)\left[\chi_{1} \gamma_{2}+\gamma_{0}^{\prime \prime} x_{0}^{\prime}(1-a)^{-1}\right] \text {, } \\
& F_{3}=f_{3}-\chi_{1}\left\{\varphi_{2}+a \gamma_{0}^{\prime \prime}\left(1-\omega^{2}\right)\left[\chi_{1} \gamma_{2}+\gamma_{0}^{\prime \prime} x_{0}^{\prime}(1-a)^{-1}\right]\right\}, \\
& \Phi_{3}=\varphi_{3}+a \gamma_{0}^{\prime \prime}\left[a \gamma_{0}^{\prime \prime} \chi_{1}\left(1-\omega^{2}\right) p_{2}-f_{2}\right] \text {, } \\
& f_{2}=x_{0}^{\prime}\left(A_{1} b^{-1} s_{21}+\dot{p}_{2} \dot{\gamma}_{2}\right)+A_{1}^{-1} \dot{p}_{2}\left[C_{1} p_{2} \dot{p}_{2}-y_{0}^{\prime}\left(2 A_{1}+b a^{-1}\right) \gamma_{2}\right]-p_{2}\left(\omega^{2} s_{11}\right. \\
& \left.+y_{0}^{\prime} a^{-1} \dot{\gamma}_{2}+z_{0}^{\prime} a^{-1} \gamma_{0}^{\prime \prime}\right)-k\left\{\gamma_{2}\left[\left(1-C_{1}\right) \dot{p}_{2} \dot{\gamma}_{2}+A_{1}\left(1+B_{1}\right) s_{21}\right]-A_{1} p_{2}\left(\gamma_{0}^{\prime \prime 2}-\dot{\gamma}_{2}^{2}\right)\right\} \text {, } \\
& \varphi_{2}=\left(1+B_{1}\right) p_{2} s_{21}+x_{0}^{\prime}\left(\dot{\gamma}_{2}^{2}+b^{-1} \gamma_{0}^{\prime \prime}\right)-A_{1}^{-1} \dot{p}_{2}\left[A_{1}^{-1} \gamma_{2} \dot{p}_{2}+\left(1-C_{1}\right) p_{2} \dot{\gamma}_{2}\right] \\
& -\gamma_{2}\left[s_{11}+z_{0}^{\prime} b^{-1} \gamma_{0}^{\prime \prime}+y_{0}^{\prime} \dot{\gamma}_{2}-k\left(C_{1} \dot{\gamma}_{2}^{2}-B_{1} \gamma_{0}^{\prime \prime 2}\right)\right] \text {, } \\
& f_{3}=-\omega^{2}\left[s_{11}\left(\chi_{1} \gamma_{2}+\frac{x_{0}^{\prime}}{1-a} \gamma_{0}^{\prime \prime}\right)+2 p_{2} s_{12}\right]+C_{1} A_{1}^{-1} \dot{p}_{2} \\
& \times\left[\dot{p}_{2}\left(\chi_{1} \gamma_{2}+\frac{x_{0}^{\prime}}{1-a} \gamma_{0}^{\prime \prime}\right)+2 p_{2} \dot{p}_{2}\left(\chi_{2} \dot{\gamma}_{2}-y_{0}^{\prime} a^{-1} \gamma_{0}^{\prime \prime}\right)\right] \\
& +x_{0}^{\prime}\left[A_{1} b^{-1} s_{22}-b A_{1}^{-1} \gamma_{0}^{\prime \prime} \dot{p}_{2}^{2}-\gamma_{0}^{\prime \prime} \dot{\gamma}_{2}\left(y_{0}^{\prime} a^{-1}-\chi_{2} \dot{\gamma}_{2}\right)\right] \\
& -y_{0}^{\prime} a^{-1} \gamma_{0}^{\prime \prime}\left[\dot{\gamma}_{2}\left(\chi_{1} \gamma_{2}+\frac{x_{0}^{\prime}}{1-a}\right)-b A_{1}^{-1} p_{2} \dot{p}_{2}\right] \\
& +z_{0}^{\prime} a^{-1}\left[\frac{1}{2} b^{-1}(2 b-1) s_{11} \gamma_{2}-\gamma_{0}^{\prime \prime}\left(\chi_{1} \gamma_{2}+\frac{x_{0}^{\prime}}{1-a}\right)-p_{2} s_{21}\right] \\
& +y_{0}^{\prime}(2-b)(1-b)^{-1}\left[\gamma_{2}\left(y_{0}^{\prime} a^{-1} \gamma_{0}^{\prime \prime}-\chi_{2} \dot{\gamma}_{2}\right)-a \gamma_{0}^{\prime \prime} p_{2} \dot{p}_{2}\right] \\
& +k\left\{\left(1-C_{1}\right)\left[\left(y_{0}^{\prime} a^{-1} \gamma_{0}^{\prime \prime}-\chi_{2} \dot{\gamma}_{2}\right) \gamma_{2} \dot{\gamma}_{2}-a \gamma_{0}^{\prime \prime} p_{2} \dot{p}_{2} \dot{\gamma}_{2}+b A_{1}^{-1} \gamma_{0}^{\prime \prime} \gamma_{2} \dot{p}_{2}^{2}\right]\right. \\
& +p_{2} \gamma_{0}^{\prime \prime}\left[2 b \dot{p}_{2} \dot{\gamma}_{2}-a A_{1}\left(1+B_{1}\right) s_{21}+2 A_{1} s_{21}\right] \\
& \left.+A_{1}\left[\left(\chi_{1} \gamma_{2}+\gamma_{0}^{\prime \prime} \frac{x_{0}^{\prime}}{1-a}\right)\left(\gamma_{0}^{\prime \prime 2}-\dot{\gamma}_{2}^{2}\right)-\left(1+B_{1}\right) \gamma_{2} s_{22}\right]\right\} \text {, } \\
& \varphi_{3}=-2 s_{12} \gamma_{2}-a \gamma_{0}^{\prime \prime} p_{2} s_{11}+\left(1+B_{1}\right)\left[p_{2} s_{22}+\left(\frac{x_{0}^{\prime}}{1-a} \gamma_{0}^{\prime \prime}+\chi_{1} \gamma_{2}\right) s_{21}\right] \\
& +2 x_{0}^{\prime} \gamma_{0}^{\prime \prime}\left(b^{-1} s_{21}-b A_{1}^{-1} \dot{p}_{2} \dot{\gamma}_{2}\right)-z_{0}^{\prime} b^{-1}\left(a \gamma_{0}^{\prime \prime 2} p_{2}+s_{21} \gamma_{2}\right)+A_{1}^{-1}\left(1-C_{1}\right) \\
& \times\left[b A_{1}^{-1} \gamma_{0}^{\prime \prime} p_{2} \dot{p}_{2}^{2}-\left(\frac{x_{0}^{\prime}}{1-a} \gamma_{0}^{\prime \prime}+\chi_{1} \gamma_{2}\right) \dot{p}_{2} \dot{\gamma}_{2}+b^{-1} \gamma_{0}^{\prime \prime 2} x_{0}^{\prime}+p_{2} \dot{\gamma}_{2}\left(\gamma_{0}^{\prime} a^{-1}-\chi_{2} \dot{\gamma}_{2}\right)\right] \\
& -y_{0}^{\prime} \gamma_{0}^{\prime \prime}\left(a p_{2} \dot{\gamma}_{2}-b A_{1}^{-1} \gamma_{2} \dot{p}_{2}\right)+A_{1}^{-2}\left[2 \dot{p}_{2}\left(y_{0}^{\prime} a^{-1} \gamma_{0}^{\prime \prime}-\chi_{2} \dot{\gamma}_{2}\right) \gamma_{2}-a \gamma_{0}^{\prime \prime} p_{2} \dot{p}_{2}^{2}\right] \\
& +k \gamma_{0}^{\prime \prime}\left[a p_{2}\left(C_{1} \dot{\gamma}_{2}^{2}-B_{1} \gamma_{0}^{\prime \prime 2}\right)-2 \gamma_{2}\left(b A_{1}^{-1} C_{1} \dot{p}_{2} \dot{\gamma}_{2}+B_{1} s_{21}\right)\right] \text {. }
\end{aligned}
$$

The last equation of (2) gives the first integral of the system (14) as follows [6]:

$$
\gamma_{2}^{2}+\dot{\gamma}_{2}^{2}+2 \mu^{-1} \gamma_{0}^{\prime \prime}\left(a p_{2} \gamma_{2}-b A_{1}^{-1} \dot{p}_{2} \dot{\gamma}_{2}+s_{21}\right)+\ldots=1-\gamma_{0}^{\prime \prime 2} .
$$

In the next, we will look for the periodic solutions of the system (14) under the conditions $A>B>C$ or $A<B<C$ ( $\omega^{2}$ is positive). The first condition [7] gives the slow rotation of the body about the major axis of the ellipsoid of inertia and the second gives 
a slow rotation of the body about the minor axis of the ellipsoid of inertia. We apply the large parameter method [8] to solve the autonomous system (14).

\section{The formal construction of the periodic solutions for a rational value of the natural frequency $\omega$}

We achieve the periodic solutions $p_{2}\left(\tau, \mu^{-1}\right), \dot{p}_{2}\left(\tau, \mu^{-1}\right), \gamma_{2}\left(\tau, \mu^{-1}\right), \dot{\gamma}_{2}\left(\tau, \mu^{-1}\right)$ of system (14) when:

$$
p_{2}(0,0)=\dot{p}_{2}(0,0)=\dot{\gamma}_{2}\left(0, \mu^{-1}\right)=0 .
$$

The generating system of (14) is obtained when $\mu \rightarrow \infty$ in the form:

$$
\ddot{p}_{2}^{(0)}+\omega^{2} p_{2}^{(0)}=0, \quad \ddot{\gamma}_{2}^{(0)}+\gamma_{2}^{(0)}=0 .
$$

So the periodic solutions of system (17), when the period $T_{0}=2 \pi n$, become:

$$
p_{2}^{(0)}=M_{1} \cos \omega \tau+M_{2} \sin \omega \tau, \quad \gamma_{2}^{(0)}=M_{3} \cos \tau,
$$

where $M_{i}, \quad i=1,2,3$ are constants to be determined.

Assuming the following solutions of system (14) [9]:

$$
\begin{aligned}
& p_{2}(\tau, \mu)=\tilde{M}_{1} \cos \omega \tau+\tilde{M}_{2} \sin \omega \tau+\sum_{k=2}^{\infty} \mu^{-k} G_{k}(\tau), \\
& \gamma_{2}(\tau, \mu)=\tilde{M}_{3} \cos \tau+\sum_{k=2}^{\infty} \mu^{-k} H_{k}(\tau),
\end{aligned}
$$

with a period $T\left(\mu^{-1}\right)=T_{0}+\alpha\left(\mu^{-1}\right)$ which reduces to (18) at $\mu \rightarrow \infty$. Let us define the quantities $\tilde{M}_{i}, i=1,2,3$ as follow:

$$
\tilde{M}_{i}=M_{i}+\beta_{i}\left(\mu^{-1}\right), \quad i=1,2,3,
$$

where $\beta_{i}$ are functions of $\mu^{-1}$ which represent the deviations of the initial values of $p_{2}, \dot{p}_{2}, \gamma_{2}$ for system (14) from their initial values of generating system (17) such that $\beta_{i}(0)=0$.

Let us express the initial conditions (16) by the relations:

$$
p_{2}\left(0, \mu^{-1}\right)=\tilde{M}_{1}, \quad \dot{p}_{2}\left(0, \mu^{-1}\right)=\omega \tilde{M}_{2}, \quad \gamma_{2}\left(0, \mu^{-1}\right)=\tilde{M}_{3}, \quad \dot{\gamma}_{2}\left(0, \mu^{-1}\right)=0 .
$$

We rewrite the periodic solutions (18) in the form:

$$
p_{2}^{(0)}=E \cos (\omega \tau-\varepsilon), \quad \gamma_{2}^{(0)}=M_{3} \cos \tau,
$$

where $E=\sqrt{M_{1}^{2}+M_{2}^{2}}$ and $\varepsilon=\tan ^{-1} M_{2} / M_{1}$. Using (22) and (12), we get: 


$$
\begin{aligned}
& s_{11}^{(0)}=E^{2}\left[\left(a \cos ^{2} \varepsilon-0.5\right)+b \omega^{2} A_{1}^{-2}\left(\sin ^{2} \varepsilon-0.5\right)+0.5\left(b \omega^{2} A_{1}^{-2}-a\right) \cos 2(\omega \tau-\varepsilon)\right] \\
& \quad-2 M_{3}\left[x_{0}^{\prime}(1-\cos \tau)+y_{0}^{\prime} \sin \tau\right]-0.5 k M_{3}^{2} C_{1}(1-\cos 2 \tau), \\
& s_{12}^{(0)}=a^{2} E \gamma_{0}^{\prime \prime} x_{0}^{\prime}(1-a)^{-1}[\cos \varepsilon-\cos (\omega \tau-\varepsilon)] \\
& \quad+E y_{0}^{\prime} b \gamma_{0}^{\prime \prime} A_{1}^{-1} \omega\left(1-a^{-1} A_{1}^{-1}\right)[\sin \varepsilon+\sin (\omega \tau-\varepsilon)] \\
& \quad+a E M_{3}\left(\chi_{1}+a \gamma_{0}^{\prime \prime} k\right)\{\cos \varepsilon-0.5 \cos [(\omega+1) \tau-\varepsilon]-0.5 \cos [(\omega-1) \tau-\varepsilon]\} \\
& \quad+\omega b A_{1}^{-1} E M_{3}\left(\chi_{2} A_{1}^{-1}-b k \gamma_{0}^{\prime \prime}\right)\{0.5 \cos [(\omega+1) \tau-\varepsilon]-0.5 \cos [(\omega-1) \tau-\varepsilon]\} \\
& \quad+E M_{3}\left(z_{0}^{\prime}-\gamma_{0}^{\prime \prime} k\right)\left\{a \cos \varepsilon+0.5\left(b \omega A_{1}^{-1}-a\right) \cos [(\omega-1) \tau-\varepsilon]\right. \\
& \left.\quad-0.5\left(b \omega A_{1}^{-1}+a\right) \cos [(\omega+1) \tau-\varepsilon]\right\}, \\
& s_{21}^{(0)}=E M_{3}\left\{a \cos \varepsilon+0.5\left(b \omega A_{1}^{-1}-a\right) \cos [(\omega-1) \tau-\varepsilon]\right. \\
& \left.\quad-0.5\left(b \omega A_{1}^{-1}+a\right) \cos [(\omega+1) \tau-\varepsilon]\right\}, \\
& s_{22}^{(0)}=E^{2} \gamma_{0}^{\prime \prime}\left[a^{2}\left(\cos ^{2} \varepsilon-0.5\right)+b^{2} \omega^{2} A_{1}^{-2}\left(\sin ^{2} \varepsilon-0.5\right)\right. \\
& \left.\quad-0.5\left(a^{2}-b^{2} \omega^{2} A_{1}^{-2}\right) \cos 2(\omega \tau-\varepsilon)\right]+0.5 M_{3}^{2}\left(a \chi_{1}+b A_{1}^{-1} \chi_{2}\right)(1-\cos 2 \tau) \\
& \quad+\gamma_{0}^{\prime \prime} M_{3}\left[a x_{0}^{\prime}(1-a)^{-1}(1-\cos \tau)+b y_{0}^{\prime} a^{-1} A_{1}^{-1} \sin \tau\right] .
\end{aligned}
$$

Substituting (22) and (23) into (16), we get:

$$
\begin{aligned}
& F_{2}^{(0)}=M_{1} L(\omega) \cos \omega \tau+M_{2} L(\omega) \sin \omega \tau+\ldots, \\
& \Phi_{2}^{(0)}=M_{3} N(\omega) \cos \tau+\ldots, \\
& F_{3}^{(0)}=M_{1} K(\omega) \cos \omega \tau+M_{2} K(\omega) \sin \omega \tau+\ldots,
\end{aligned}
$$

where:

$$
\begin{aligned}
& L(\omega)=\omega^{2}\left[-\left(a M_{1}^{2}+b \omega^{2} A_{1}^{-2} M_{2}^{2}\right)+0.25\left(M_{1}^{2}+M_{2}^{2}\right)\left(C_{1} A_{1}^{-1}+3 a+b \omega^{2} A_{1}^{-2}\right)\right] \\
& \quad+2 \omega^{2} M_{3} x_{0}^{\prime}-\gamma_{0}^{\prime \prime}\left[z_{0}^{\prime} a^{-1}+a \chi_{1}\left(1-\omega^{2}\right)\right]+k\left\{A_{1}\left(\gamma_{0}^{\prime \prime 2}-0.5 M_{3}^{2}\right)\right. \\
& \left.\quad+0.5 M_{3}^{2}\left[a\left(A_{1}-2 \omega^{2}\right)+\omega^{2} b\right]\right\}, \\
& N(\omega)=-\left(a M_{1}^{2}+b \omega^{2} A_{1}^{-2} M_{2}^{2}\right)-0.5\left(M_{1}^{2}+M_{2}^{2}\right)\left[a B_{1}+\omega^{2} A_{1}^{-2}(1-b)\right] \\
& \quad+2 M_{3} x_{0}^{\prime}-\gamma_{0}^{\prime \prime}\left[z_{0}^{\prime} b^{-1}-a \chi_{1}\left(1-\omega^{2}\right)\right]+k\left[M_{3}^{2}(b-a)-B_{1} \gamma_{0}^{\prime \prime 2}\right], \\
& K(\omega)=-2 \omega^{2} \gamma_{0}^{\prime \prime}\left[a^{2} x_{0}^{\prime} M_{1}(1-a)^{-1}+\omega y_{0}^{\prime} b M_{2}\left(1-a^{-1} A_{1}^{-1}\right) A_{1}^{-1}\right. \\
& \left.\quad+0.25 a^{-1} A_{1}^{-1} C_{1} y_{0}^{\prime}\left(M_{1}^{2}+M_{2}^{2}\right)\right]-2 \omega^{2} a M_{1} M_{3}\left[\chi_{1}+a \gamma_{0}^{\prime \prime} k+z_{0}^{\prime}-\gamma_{0}^{\prime \prime} k\right] \\
& \quad+a M_{1} M_{3}\left[2 A_{1} k \gamma_{0}^{\prime \prime}-z_{0}^{\prime} a^{-1}-a A_{1} k \gamma_{0}^{\prime \prime}\left(1+B_{1}\right)-\chi_{1}\left(1+B_{1}\right)\right] .
\end{aligned}
$$

Using (24) and (25), the following functions are obtained:

$$
\begin{array}{ll}
g_{2}\left(T_{0}\right)=-\pi n \omega^{-1} M_{2} L(\omega), & \dot{g}_{2}\left(T_{0}\right)=\pi n M_{1} L(\omega), \\
h_{2}\left(T_{0}\right)=0, & \dot{h}_{2}\left(T_{0}\right)=\pi n M_{3} N(\omega), \\
g_{3}\left(T_{0}\right)=-\pi n \omega^{-1} M_{2} K(\omega), & \dot{g}_{3}\left(T_{0}\right)=\pi n M_{1} K(\omega) .
\end{array}
$$

Substituting by the initial conditions (21) into the first integration (17) when $\tau=0$, we get:

$$
M_{3}^{2}+2 M_{3} \beta_{3}+\beta_{3}^{2}+2 \mu^{-1} a \gamma_{0}^{\prime \prime} M_{3}\left(M_{1}+\beta_{1}\right)=1-\gamma_{0}^{\prime \prime 2} .
$$

Let $\gamma_{0}^{\prime \prime}$ depends on $\mu^{-1}$, we get: 


$$
\gamma_{0}^{\prime \prime}=\mu^{-1} \Gamma, \quad 0<\Gamma<1
$$

Taking into consideration, Eqs. (27) and (28), we get $M_{3}, \beta_{3}$ as follows:

$$
M_{3}=1, \quad \beta_{3}=-a \Gamma \mu^{-2} \tilde{M}_{1}-\frac{1}{2} \mu^{-2} \Gamma^{2}+\ldots
$$

The independent conditions for periodicity are:

$$
\begin{aligned}
& -\left(L_{1}(\omega)-\omega^{2} N_{1}(\omega)\right) \pi n \omega^{-1} \tilde{M}_{2}+\mu^{-1} G_{3}\left(T_{0}\right)+\ldots=0 \\
& \left(L_{1}(\omega)-\omega^{2} N_{1}(\omega)\right) \pi n \tilde{M}_{1}+\mu^{-1} \dot{G}_{3}\left(T_{0}\right)+\ldots=0 \\
& \mu^{-2}\left(\dot{H}_{2}\left(T_{0}\right)+\mu^{-1} \dot{H}_{3}\left(T_{0}\right)\right) \tilde{M}_{3}^{-1}+\ldots=\alpha\left(\mu^{-1}\right)
\end{aligned}
$$

where $L_{1}(\omega), N_{1}(\omega)$ are obtained from $L(\omega), N(\omega)$ replacing $M_{i}$ by $\left(M_{i}+\beta_{i}\right), i=1,2,3$ to get:

$$
L_{1}(\omega)-\omega^{2} N_{1}(\omega)=W_{0}(\omega)\left(\tilde{M}_{1}^{2}+\tilde{M}_{2}^{2}\right)-\gamma_{0}^{\prime \prime}\left[z_{0}^{\prime} W_{1}(\omega)+k \gamma_{0}^{\prime \prime} W_{2}(\omega)\right]-k W_{3}(\omega) \tilde{M}_{3}^{2},
$$

where:

$$
\begin{array}{ll}
W_{0}(\omega)=(a-1)(a+b-2) / 2 b, & W_{1}(\omega)=[3(a+b)-2(2 a b+1)] / a b \\
W_{2}(\omega)=2 \omega^{2}[1-(a+b)], & W_{3}(\omega)=\omega^{2} b
\end{array}
$$

For zeros approximation for power series of $1 / \mu$, Eq. (30) give:

$$
M_{1}=M_{2}=0 \text {. }
$$

Since the $z$-axis is directed along with the major or the minor axis of the ellipsoid of inertia of the body, we get: $W_{0}(\omega)>0$ for all $\omega$ under consideration.

Assume that:

$$
\gamma_{0}^{\prime \prime}\left[z_{0}^{\prime} W_{1}+k \gamma_{0}^{\prime \prime} W_{2}\right]+k W_{3}(\omega) M_{3}^{2} \neq 0
$$

Using (30), we get $\beta_{1}, \beta_{2}$ in power series expansions of powers less than $\mu^{-2}$. Then for the rational values of the natural frequency $\omega$ does not equal to $(1,2,1 / 2,3,1 / 3, \ldots)$, we get the required periodic solutions and the correction of the period $\alpha\left(\mu^{-1}\right)$ as:

$$
\begin{aligned}
& p_{1}\left(\tau, \mu^{-1}\right)=\mu^{-1}\left[x_{0}^{\prime}(1-a)^{-1} \gamma_{0}^{\prime \prime}+\chi_{1} M_{3} \cos \tau\right]+\ldots, \\
& q_{1}\left(\tau, \mu^{-1}\right)=\mu^{-1}\left[y_{0}^{\prime}(1-b)^{-1} \gamma_{0}^{\prime \prime}+A_{1}^{-1} M_{3} \chi_{2} \sin \tau\right]+\ldots, \\
& r_{1}\left(\tau, \mu^{-1}\right)=1-0.25 \mu^{-2} M_{3}\left[k M_{3} C_{1}+4 x_{0}^{\prime}(1-\cos \tau)+y_{0}^{\prime} \sin \tau-k M_{3} C_{1} \cos 2 \tau\right]+\ldots, \\
& \gamma\left(\tau, \mu^{-1}\right)=M_{3} \cos \tau-0.5 \mu^{-2} \Gamma^{2} \cos \tau+\ldots, \\
& \gamma^{\prime}\left(\tau, \mu^{-1}\right)=-M_{3} \sin \tau+0.5 \mu^{-2} \Gamma^{2} \sin \tau+\ldots, \\
& \gamma^{\prime \prime}\left(\tau, \mu^{-1}\right)=\gamma_{0}^{\prime \prime}+\mu^{-2} M_{3}\left[x_{0}^{\prime}(1-a)^{-1} \gamma_{0}^{\prime \prime}-0.5 M_{3} C_{1}\left(\frac{z_{0}^{\prime}}{a+b-1}+0.5 k \gamma_{0}^{\prime \prime}\right)\right. \\
& \left.\quad-x_{0}^{\prime}(1-a)^{-1} \gamma_{0}^{\prime \prime} \cos \tau+y_{0}^{\prime}(1-b)^{-1} \gamma_{0}^{\prime \prime} \sin \tau+0.25 M_{3} C_{1}\left(\frac{2 z_{0}^{\prime}}{a+b-1}+k \gamma_{0}^{\prime \prime}\right) \cos 2 \tau\right]+\ldots,
\end{aligned}
$$




$$
\alpha\left(\mu^{-1}\right)=2 \mu^{-2} \pi n\left\{M_{3} x_{0}^{\prime}-z_{0}^{\prime} \gamma_{0}^{\prime \prime}-0.5 k\left[\gamma_{0}^{\prime \prime 2}\left(b B_{1}-a A_{1}\right)+B_{1} \gamma_{0}^{\prime \prime}\left(1-\gamma_{0}^{\prime \prime}\right)-0.125 C_{1} M_{3}^{2}\right]\right\}+\ldots
$$

The obtained solutions (35) and (36) are considered as the generalization of the corresponding problem in gravity field which studied in previous works [10] (when $\mathrm{k}=0$ ), the deviations between them are given by:

$$
\begin{aligned}
& \Delta p_{1}=\mu^{-1}\left(1-\omega^{2}\right)^{-1} k M_{3} \gamma_{0}^{\prime \prime}\left(A_{1}-\omega^{2}\right) \cos \tau+\ldots, \\
& \Delta q_{1}=\mu^{-1} A_{1}^{-1} M_{3}\left\{\left(1-\omega^{2}\right)^{-1}\left[k\left(A_{1}-\omega^{2}\right) \gamma_{0}^{\prime \prime}\right]-k A_{1} \gamma_{0}^{\prime \prime}\right\} \sin \tau+\ldots, \\
& \Delta r_{1}=-0.25 \mu^{-2} M_{3} k C_{1}(1-\cos 2 \tau)+\ldots, \\
& \Delta \gamma=\mu^{-2}[0]+\ldots, \quad \Delta \gamma^{\prime}=\mu^{-2}[0]+\ldots, \\
& \Delta \gamma^{\prime \prime}=-0.25 \mu^{-2} M_{3}^{2} C_{1} k \gamma_{0}^{\prime \prime}(1-\cos 2 \tau)+\ldots, \\
& \Delta \alpha\left(\mu^{-1}\right)=-\mu^{-2} \pi n k\left[\gamma_{0}^{\prime \prime 2}\left(b B_{1}-a A_{1}\right)-0.5 M_{3}^{2}(b-a)+B_{1} \gamma_{0}^{\prime \prime}\left(1-\gamma_{0}^{\prime \prime}\right)\right]+\ldots .
\end{aligned}
$$

\section{Geometric interpretation of the motion}

The geometric interpretation for the motion of the body at any instant of time to Euler's angles definitions $\theta, \psi, \phi$ is given by [11]:

$$
\begin{aligned}
& \theta=\theta_{0}+\mu^{-2} \operatorname{cosec} \theta_{0}\left[\theta_{2}\left(t+t_{0}\right)-\theta_{2}\left(t_{0}\right)\right]+\ldots \\
& \psi=\psi_{0}+0.5 M g \ell C^{-1} r_{0}^{-1}\left(\chi_{1}-\chi_{2} A_{1}^{-1}\right) t+\mu^{-1} \sqrt{M g \ell / C}\left[\psi_{1}\left(t+t_{0}\right)-\psi_{1}\left(t_{0}\right)\right]+\ldots \\
& \phi=\phi_{0}+\left[r_{0}^{-1}-0.5 M g \ell C^{-1} r_{0}^{-1} \cos \theta_{0}\left(\chi_{1}-\chi_{2} A_{1}^{-1}\right)\right] t+\ldots
\end{aligned}
$$

where:

$$
\begin{aligned}
\theta_{2}(t)= & M_{3}\left[y_{0}^{\prime} \gamma_{0}^{\prime \prime}(1-b)^{-1} \sin r_{0}^{-1} t-x_{0}^{\prime} \gamma_{0}^{\prime \prime}(1-a)^{-1} \cos r_{0}^{-1} t\right. \\
& \left.+0.5 C_{1}\left(\frac{z_{0}^{\prime}}{a+b-1}-0.667 k \gamma_{0}^{\prime \prime}\right) \cos 2 r_{0}^{-1} t\right] \\
& \psi_{1}(t)=0.25\left(\chi_{1}+\chi_{2} A_{1}^{-1}\right)+\sin 2 r_{0}^{-1} t+y_{0}^{\prime} \gamma_{0}^{\prime \prime}(1-b)^{-1} \cos r_{0}^{-1} t+x_{0}^{\prime}(1-a)^{-1} \gamma_{0}^{\prime \prime} \sin r_{0}^{-1} t
\end{aligned}
$$

\section{Numerical solutions}

In this section, we use a computer program to determine the obtained solutions (19) and their derivatives for the time in the interval $t \in[0,300]$. On the other hand, we use the fourth-order Runge-Kutta method [12] through another program to obtain numerical solutions for the autonomous system (14). In the end, we compare both solutions to check the accuracy of the method of solutions. These results are obtained through Tables 1 and 2. From these Tables, we deduce that the numerical solutions are in agreement with the analytical ones which prove the accuracy of considered methods. 


\section{Conclusions}

We conclude that the problem of the motion of a rigid body about a fixed point is studied in many works [13-18] in both the uniform and gravity fields. We study our problem in case of a right angle of nutation $\theta_{0}$ when its center of mass does not necessarily coincide with the fixed point. The equations of motions of the problem are obtained and reduced to a quasilinear autonomous system. The obtained system is solved by assuming a large parameter achieved from an angular velocity component tends to zero. The obtained solutions are treated through computer programs in a bounded interval of time. The autonomous system is treated with the Runge-Kutta method in the same interval of time to obtain the numerical solutions of the motion. Both obtained solutions are in full agreement with others which prove the accuracy of both numerical and analytical techniques used in solving the problem. For the geometric interpretation obtained, we note that:

\section{The precession angle $\psi$}

From (38) when $\mu \rightarrow \infty$, we deduce that the precession angle $\psi$ is sufficiently large because $r_{0}$ is sufficiently small, that is, we obtain a case of large precession $\psi=\psi_{0}+0.5 M g \ell C^{-1} r_{0}^{-1}\left(\chi_{1}-\chi_{2} A_{1}^{-1}\right) t$.

\section{The nutation angle $\theta$}

We obtain a case of steady regular permutation: $\theta=\theta_{0}$.

\section{The pure rotation angle $\phi$}

The case of a large pure rotation is obtained which depends on $1 / r_{0}$ in the form:

$$
\phi=\phi_{0}+\left[r_{0}-0.5 M g \ell C^{-1} r_{0}^{-1} \cos \theta_{0}\left(\chi_{1}-\chi_{2} A_{1}^{-1}\right)\right] t .
$$

The large parameter technique used here is considered as the only one suitable for this problem in the origin domain of $r_{\mathrm{o}}$ tends to zero. Poincaré-Lindstedt method or Krylov Boboliubov Mitropolski one is failed to solve this problem because they depend on achieving a small parameter in domain $r_{0}$ tends to infinity. We conduct a comparison of the results of this manuscript with the results of the previous work. The results were obtained in [19] deals with the disk problem which satisfies the symmetry moments of inertia about two principal axes of the ellipsoid of inertia but our results here treat the general rigid problem in a limiting value of the Euler's angle $\theta_{o} \approx \pi / 2$. The advantage of our used technique [20] depends on a large parameter $\mu \rightarrow \infty$. The obtained solutions are checked using two programs to assert their accuracy through Tables 1 and 2. The main results in our work are the obtained analytical solutions in Eq. (35) which is represented through computerized digital data in Table 1 . The secondary results are proving the validity of these solutions which are given through the Runge-Kutta method in 
Table 1 Represents the values of the obtained analytical solutions using the large parameter method in the interval $t \in[0,300]$

\begin{tabular}{|c|c|c|c|c|}
\hline$t$ & $p_{2 a}$ & $\gamma_{2 a}$ & $x_{a}=d p_{2 a} / d t$ & $y_{a}=d \gamma_{2 a} / d t$ \\
\hline 0 & $1.88117 \mathrm{E}-15$ & 1 & 0 & 0 \\
\hline 10 & $1.52189 \mathrm{E}-15$ & 0.809017 & $-1.10572 E-15$ & -0.587785 \\
\hline 20 & $5.81312 \mathrm{E}-16$ & 0.309017 & $-1.78909 E-15$ & -0.951057 \\
\hline 30 & $-5.81312 E-16$ & -0.309017 & $-1.78909 E-15$ & -0.951056 \\
\hline 40 & $-1.5219 E-15$ & -0.809017 & $-1.10572 E-15$ & -0.587785 \\
\hline 50 & $-1.88117 E-15$ & -1 & $-2.84048 \mathrm{E}-22$ & $-1.50996 \mathrm{E}-07$ \\
\hline 60 & $-1.52189 E-15$ & -0.809017 & $1.10572 \mathrm{E}-15$ & 0.587785 \\
\hline 70 & $-5.81312 \mathrm{E}-16$ & -0.309017 & $1.78909 \mathrm{E}-15$ & 0.951056 \\
\hline 80 & $5.81312 \mathrm{E}-16$ & 0.309017 & $1.78909 E-15$ & 0.951056 \\
\hline 90 & $1.5219 \mathrm{E}-15$ & 0.809017 & $1.10572 E-15$ & 0.587785 \\
\hline 100 & $1.88117 \mathrm{E}-15$ & 1 & $5.68096 \mathrm{E}-22$ & 3.01992E-07 \\
\hline 110 & $1.52189 \mathrm{E}-15$ & 0.809017 & $-1.10572 \mathrm{E}-15$ & -0.587785 \\
\hline 120 & $5.81312 \mathrm{E}-16$ & 0.309017 & $-1.78909 E-15$ & -0.951057 \\
\hline 130 & $-5.81313 \mathrm{E}-16$ & -0.309017 & $-1.78909 \mathrm{E}-15$ & -0.951056 \\
\hline 140 & $-1.52189 E-15$ & -0.809017 & $-1.10572 \mathrm{E}-15$ & -0.587785 \\
\hline 150 & $-1.88117 E-15$ & -1 & $4.48654 \mathrm{E}-23$ & $2.38498 \mathrm{E}-08$ \\
\hline 160 & $-1.52189 E-15$ & -0.809017 & $1.10572 E-15$ & 0.587785 \\
\hline 170 & $-5.81311 E-16$ & -0.309016 & $1.78909 E-15$ & 0.951057 \\
\hline 180 & $5.81314 \mathrm{E}-16$ & 0.309018 & $1.78909 E-15$ & 0.951056 \\
\hline 190 & 1.5219E-15 & 0.809017 & $1.10572 \mathrm{E}-15$ & 0.587785 \\
\hline 200 & $1.88117 \mathrm{E}-15$ & 1 & $1.13619 E-21$ & $6.03983 \mathrm{E}-07$ \\
\hline 210 & $1.5219 E-15$ & 0.809017 & $-1.10572 \mathrm{E}-15$ & -0.587785 \\
\hline 220 & $5.81312 \mathrm{E}-16$ & 0.309017 & $-1.78909 \mathrm{E}-15$ & -0.951056 \\
\hline 230 & $-5.81312 E-16$ & -0.309017 & $-1.78909 E-15$ & -0.951056 \\
\hline 240 & $-1.5219 \mathrm{E}-15$ & -0.809017 & $-1.10572 E-15$ & -0.587785 \\
\hline 250 & $-1.88117 E-15$ & -1 & $1.27079 E-21$ & $6.75532 \mathrm{E}-07$ \\
\hline 260 & $-1.52189 E-15$ & -0.809016 & $1.10572 \mathrm{E}-15$ & 0.587786 \\
\hline 270 & $-5.81313 \mathrm{E}-16$ & -0.309018 & $1.78909 \mathrm{E}-15$ & 0.951056 \\
\hline 280 & $5.81311 \mathrm{E}-16$ & 0.309017 & $1.78909 \mathrm{E}-15$ & 0.951057 \\
\hline 290 & $1.52189 \mathrm{E}-15$ & 0.809017 & $1.10572 \mathrm{E}-15$ & 0.587785 \\
\hline 300 & $1.88117 \mathrm{E}-15$ & 1 & $-8.97307 \mathrm{E}-23$ & $-4.76995 \mathrm{E}-08$ \\
\hline
\end{tabular}

Table 2. So we confirm that the presented numerical results would correlate to results obtained by other distinguished numerical techniques (except the Runge-Kutta method) will reveal the same results obtained in Table 1. 
Table 2 represents the values of the numerical solutions using fourth-order Runge-Kutta method in the interval $t \in[0,300]$

\begin{tabular}{|c|c|c|c|c|}
\hline$t$ & $p_{2 n}$ & $\gamma_{2 n}$ & $x_{n}=d p_{2 n} / d t$ & $y_{n}=d \gamma_{2 n} / d t$ \\
\hline 0 & $1.8812 \mathrm{E}-15$ & 1 & 0 & 0 \\
\hline 10 & $1.5247 \mathrm{E}-15$ & 0.809035 & $-1.09756 \mathrm{E}-15$ & -0.587738 \\
\hline 20 & $5.9028 \mathrm{E}-16$ & 0.309102 & $-1.77911 \mathrm{E}-15$ & -0.951001 \\
\hline 30 & $-5.6779 \mathrm{E}-16$ & -0.308864 & $-1.78633 E-15$ & -0.951064 \\
\hline 40 & $-1.5106 E-15$ & -0.808859 & $-1.11651 E-15$ & -0.587913 \\
\hline 50 & $-1.8809 E-15$ & -0.999934 & $-2.35325 E-17$ & -0.000245929 \\
\hline 60 & $-1.5383 E-15$ & -0.809126 & $1.07833 \mathrm{E}-15$ & 0.5875 \\
\hline 70 & $-6.1263 E-16$ & -0.309316 & $1.77147 \mathrm{E}-15$ & 0.950862 \\
\hline 80 & $5.4519 \mathrm{E}-16$ & 0.30861 & $1.79318 \mathrm{E}-15$ & 0.951078 \\
\hline 90 & $1.4963 \mathrm{E}-15$ & 0.808661 & $1.13524 \mathrm{E}-15$ & 0.588073 \\
\hline 100 & $1.8803 \mathrm{E}-15$ & 0.999868 & $4.70583 \mathrm{E}-17$ & 0.000491738 \\
\hline 110 & $1.5517 \mathrm{E}-15$ & 0.809217 & $-1.05894 E-15$ & -0.587262 \\
\hline 120 & $6.3487 \mathrm{E}-16$ & 0.309529 & $-1.76355 E-15$ & -0.950724 \\
\hline 130 & $-5.225 \mathrm{E}-16$ & -0.308356 & $-1.79974 \mathrm{E}-15$ & -0.951091 \\
\hline 140 & $-1.4818 \mathrm{E}-15$ & -0.808463 & $-1.1538 \mathrm{E}-15$ & -0.588234 \\
\hline 150 & $-1.8795 E-15$ & -0.999802 & $-7.05736 \mathrm{E}-17$ & -0.000737578 \\
\hline 160 & $-1.5648 E-15$ & -0.809309 & $1.03937 E-15$ & 0.587025 \\
\hline 170 & $-6.5702 E-16$ & -0.309743 & $1.75536 \mathrm{E}-15$ & 0.950585 \\
\hline 180 & 4.9974E-16 & 0.308102 & $1.80602 \mathrm{E}-15$ & 0.951104 \\
\hline 190 & $1.4671 \mathrm{E}-15$ & 0.808265 & 1.17217E-15 & 0.588394 \\
\hline 200 & $1.8783 \mathrm{E}-15$ & 0.999736 & $9.40748 \mathrm{E}-17$ & 0.000983447 \\
\hline 210 & $1.5777 \mathrm{E}-15$ & 0.809399 & $-1.01965 E-15$ & -0.586787 \\
\hline 220 & $6.7906 \mathrm{E}-16$ & 0.309956 & $-1.74689 \mathrm{E}-15$ & -0.950446 \\
\hline 230 & $-4.7689 \mathrm{E}-16$ & -0.307848 & $-1.81201 E-15$ & -0.951117 \\
\hline 240 & $-1.4521 \mathrm{E}-15$ & -0.808067 & $-1.19036 \mathrm{E}-15$ & -0.588553 \\
\hline 250 & $-1.8769 E-15$ & -0.99967 & $-1.17558 E-16$ & -0.00122914 \\
\hline 260 & $-1.5903 E-15$ & -0.80949 & $9.99769 \mathrm{E}-16$ & 0.586549 \\
\hline 270 & $-7.0098 \mathrm{E}-16$ & -0.310169 & $1.73814 \mathrm{E}-15$ & 0.950307 \\
\hline 280 & $4.5398 \mathrm{E}-16$ & 0.307593 & $1.81772 \mathrm{E}-15$ & 0.95113 \\
\hline 290 & $1.4369 \mathrm{E}-15$ & 0.807869 & $1.20835 E-15$ & 0.588713 \\
\hline 300 & $1.8751 \mathrm{E}-15$ & 0.999603 & $1.41019 \mathrm{E}-16$ & 0.00147495 \\
\hline
\end{tabular}

Acknowledgements

Not applicable.

Authors' contributions

I am the individual authors of the manuscript.

Funding

There is no funding for the research reported

Availability of data and materials

Data sharing not applicable to this article as no datasets were generated or analyzed during the current study.

Competing interests

The author declares that he has no competing interests.

Author details

${ }^{1}$ Mechanical Engineering Department, College of Engineering and Islamic Architecture, Umm Al-Qura University, P. O. Box 5555, Makkah, Saudi Arabia. ${ }^{2}$ Mathematics Department, Faculty of Science, Tanta University, P.O. Box 31527, Tanta, Egypt.

Received: 16 July 2020 Accepted: 23 December 2020

Published online: 14 January 2021 


\section{References}

1. Mc Hugh, J.A.: A historical survey of ordinary linear differential equations with a large parameter and turning points. Arch. Hist. Exact Sci. 7(4), 277-324 (1971)

2. Moiseev, N.N.: Asymptotic Methods of Nonlinear Mechanics. Moscow, Nauka 400 (1981)

3. Shkil', M.: On asymptotic methods in the theory of differential equations of Mathematical physics. J. Nonlinear Math. Phys. 3(1-2), 40-50 (1996)

4. El-Barki, F.A., Ismail, A.I.: Limiting case for the motion of a rigid body about a fixed point in the Newtonian force field. ZAMM 75(12), 821-829 (1995). https://doi.org/10.1002/zamm.19950751203

5. Sartabanov, Z.A, Omarova, B.Z.: On multi-periodic solutions of quasilinear autonomous systems with an operator of differentiation on the Lyapunov's vector field. Bulletin of Karaganda University, Section "Mathematics" 2(94), 70-81 (2019)

6. Amer, T.S., Amer, W.S.: Substantial condition for the fourth first integral of the rigid body problem. Math. Mech. Solids 23(8), 1237-1246 (2018)

7. Amer, W.S.: The necessary and sufficient condition for the stability of a rigid body. J. Adv. Phys. 13(6), 4999 (2017)

8. Nayfeh, A.H.: Introduction to Perturbation Technique, pp. 360-364. Wiley, Weinheim (2011)

9. Amer, T.S.: The rotational motion of the electromagnetic symmetric rigid body. Appl. Math. Inf. Sci. 10(4), 1453-1464 (2016)

10. Yehia, H.M.: On the regular precession of an asymmetric rigid body acted upon by uniform gravity and magnetic fields. Egypt. J. Basic Appl. Sci. 2(3), 200-205 (2015). https://doi.org/10.1016/j.ejbas.2015.03.002

11. Amer, T.S., Abady, I.M.: On the application of the KBM method for the 3-D motion of asymmetric rigid body. Nonlinear Dyn. 89, 1591-1609 (2017). https://doi.org/10.1007/s11071-017-3537-7

12. Vitoriano, R.: Numerical Methods for Partial Differential Equations: An Introduction. Wiley, Hoboken (2016)

13. Chernousko, F.L., Akulenko, L.D., Leshchenko, D.D.: Evolution of motions of a rigid body about its center of mass, pp. 1-12. Springer, Berlin (2017)

14. Scarpello, G.M., Rotelli, D.: Motions about a fixed point by hypergeometric functions: new non-complex analytical solutions and integration of the herpolhode. Celest. Mech. Dyn. Astron. 130, 42 (2018)

15. Borisov, A.V., Mamaev, I.S.: Rigid Body Dynamics. Higher Education Press, Berlin (2018)

16. Ismail, A.I., Amer, T.S.: The fast spinning motion of a rigid body in the presence of a gyrostatic momentum $\ell 3$. Acta Mech. 154, 31-46 (2002)

17. Ismail, A.I.: On the motion of a rigid body in a Newtonian field of force exerted by three attracting centers. ASCE 21(1), 67-77 (2010)

18. Ershkov, S.V., Christianto, V., Shamin, R.V., Giniyatullin, A.R.: About analytical ansatz to the solving procedure for Kelvin-Kirchhoff equations. Eur. J. Mech. B Fluids 79C, 87-91 (2020)

19. Ismail, A.I.: Applying the large parameter technique for solving a slow rotary motion of a disc about a fixed point. Int. J. Aerosp. Eng. 2020, 8854136 (2020)

20. Ismail, A.I.: Solving a problem of rotary motion for a heavy solid using the large parameter method. Adv. Astron. 2020, 2764867 (2020)

\section{Publisher's Note}

Springer Nature remains neutral with regard to jurisdictional claims in published maps and institutional affiliations.

\section{Submit your manuscript to a SpringerOpen ${ }^{\circ}$ journal and benefit from:}

- Convenient online submission

- Rigorous peer review

- Open access: articles freely available online

- High visibility within the field

- Retaining the copyright to your article

Submit your next manuscript at $\gg$ springeropen.com 